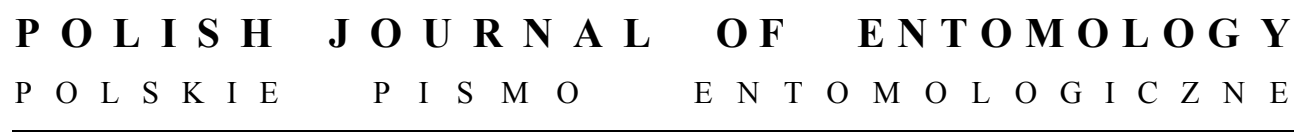

VOL. 80: $343-364$

Gdynia

30 June 2011

DOI: $10.2478 / \mathrm{v} 10200-011-0024-1$

\title{
Faunistic review of Polish Platypodinae and Scolytinae (Coleoptera: Curculionidae)
}

\author{
TOMASZ MOKRZYCKI ${ }^{1}$, JACEK HILSZCZAŃSKI ${ }^{2}$, JERZY BOROWSKI ${ }^{3}$, \\ RAFAŁ CIEŚLAK ${ }^{4}$, ANDRZEJ MAZUR ${ }^{5}$, MAREK MIŁKOWSKI $^{6}$, HENRYK SZOŁTYS ${ }^{7}$ \\ ${ }^{1}$ Department of Forest Protection and Ecology, Warsaw University of Life Sciences, \\ Nowoursynowska 159/34,02-776 Warszawa, Poland, e-mail:tomasz_mokrzycki@sggw.pl; \\ ${ }^{2}$ Department of Forest Protection, Forest Research Institute, Sękocin Stary, Braci Leśnej 3, 05-090 \\ Raszyn; ${ }^{3}$ Institute of Forest Sciences, University of Łódź, Branch in Tomaszów Mazowiecki, \\ Konstytucji 3 Maja 65/67, 97-200 Tomaszów Mazowiecki; ${ }^{4}$ Lotnicza 2/4, 21-040 Świdnik; \\ ${ }^{5}$ Department of Forest Entomology, Poznań University of Life Sciences, Wojska Polskiego 28 , \\ 60-637 Poznań; ${ }^{6}$ Królowej Jadwigi 19/21, 26-600 Radom; ${ }^{7}$ Park 9, 42-690 Brynek
}

\begin{abstract}
Based on a survey of the literature and collections, the distributions of the Platypodinae and Scolytinae (Curculionidae) within the present-day borders of Poland are summarized. An updated and annotated checklist is given of Polish pinhole borers (1 genus, 1 species) and bark beetles (35 genera, 110 species). Carphoborus cholodkovskyi SPESSIVTSEV has been removed from the list; the presence of Hylastinus obscurus (MARSHAM), Pteleobius kraatzii (EICHHOFF), Pityophthorus exsculptus (RATZEBURG), Cryphalus saltuarius WeISE, Thamnurgus kaltenbachii (BACH), T. varipes EICHHOFF, Pityogenes irkutensis monacensis FUCHS, P. saalasi Eggers, Xyleborus eurygraphus (RATZEBURG) and X. pfeilii (RATZEBURG), reported by previous authors, needs to be confirmed by new findings. Pitophthorus carniolicus WichmanN, Xylosandrus germanus (BLANDFORD) and Crypturgus subcribrosus EGGERS were recorded generally from Poland. Hylastes plumbeus BLANDFORD, Phloeosinus aubei (PERRIS), Gnathotrichus materiarius (FITCH), Orthotomicus erosus (WOLLASTON), Scolytus sulcifrons REY and Trypodendron laeve EGGERS (KNÍŽEK 2011) were erroneously recorded from Poland.
\end{abstract}

KEY WORDS: Coleoptera, Curculionidae, Platypodinae, Scolytinae, Poland.

\section{INTRODUCTION}

Platypodinae and Scolytinae are a group of weevils. Platypodinae live in nutritional symbiosis with ambrosia fungi. The beetles excavate tunnels in trees in which they cultivate fungal gardens, their sole source of nutrition. Scolytinae reproduce in the inner bark (living 
and dead phloem tissues) of trees. Some bark beetles form a symbiotic relationship with certain fungi. Other species develop in seeds and on herbaceous plants (SCHEDL 1972, WOOD 1986). Bark beetles and pinhole borers are a relatively well-known group of Coleoptera in Poland. However, information about the distribution of individual species in the country is incomplete. Ten species were recorded in the country more than fifty years ago. The existing checklists of Platypodinae and Scolytinae in Poland (MROCZKOWSKI \& StEFAŃSKa 1991, WANAT \& MOKRZYCKI 2005, KNiŽEK 2011) require amendment. The present paper summarizes and comments on available literature records, and provides additional findings of Platypodinae and Scolytinae.

\section{Acknowledgements}

The authors thank Tadeusz Bziuk and Dawid Marczak for their data on new localities of bark beetles in Poland.

\section{MATERIALS}

The data presented below are based on a literature survey as well as specimens deposited in the Department of Forest Protection and Ecology, Warsaw University of Life Sciences (DFPEW), Warsaw, the private collections of Rafał Cieślak, Świdnik, Poland (PCRC), Jacek Hilszczański, Warsaw, Poland (PCJH), Dawid Marczak, Izabelin, Poland (PCDM), Andrzej Mazur, Poznań, Poland (PCAM), Marek Miłkowski, Radom, Poland (PCMM), Tomasz Mokrzycki, Warszawa, Poland (PCTM), Rafał Ruta, Wrocław, Poland (PCRR) and Henryk Szołtys, Brynek, Poland (PCHS).

The abbreviations used in the text are as follows: for. insp. - forest inspectorate; nat. res. - natural reserve.

\section{ANNOTATED CHECKLIST OF PLATYPODINAE AND SCOLYTINAE}

\section{Platypodinae}

\section{Platypus cylindrus (FABRICIUS, 1792)}

\section{New records}

Wielkopolska-Kujawy Lowland: Krotoszyn for. insp., (XT73), VI-VII 2007, IBL2 traps, 3 exx. leg. J. Hilszczański (PCJH), Upper Silesia: Rudziniec (CA18), 6-13 VII 1995, 4 exx., leg. R. Moś (PCTM); Małopolska Upland: Spała (DC41), 15-16 IV 2011, 27 exx, leg. J. Borowski (DFPEW) and Sandomierz Lowland: Sobów near Tarnobrzeg (EB50), 4 VI 2000, 3 exx., leg. R. Cieślak (PCTM).

Previously recorded from: Baltic Coast, Masurian Lake District, Wielkopolska-Kujawy Lowland, Mazovian Lowland, Białowieża Primeval Forest, Lower Silesia, Trzebnica Hills, 
Upper Silesia, Kraków-Wieluń Upland, Małopolska Upland, Lublin Upland, Roztocze, Eastern Sudetes Mts., Western Beskid Mts., Eastern Beskid Mts. (BURAKOWSKI et al. 1992, MOKRZYCKI et al. 2008).

\section{Scolytinae}

\section{Hylastes angustatus (HERBST, 1793)}

Known from: Baltic Coast, Wielkopolska-Kujawy Lowland, Mazovian Lowland, Białowieża Primeval Forest, Lower Silesia, Upper Silesia, Kraków-Wieluń Upland, Małopolska Upland, Świętokrzyskie Mts., Lublin Upland, Roztocze, Sandomierz Lowland, Eastern Sudetes Mts., Western Beskid Mts., Eastern Beskid Mts. (BURAKOWSKI et al. 1992).

Hylastes ater (PAYKULL, 1800)

Recorded in the whole of Poland (BURAKOWSKI et al. 1992).

\section{Hylastes attenuatus ERICHSON, 1836}

Recorded throughout Poland (BURAKOWSKI et al. 1992).

\section{Hylastes brunneus ERICHSON, 1836}

Recorded from: Wielkopolska-Kujawy Lowland, Białowieża Primeval Forest, Lower Silesia, Roztocze, Western Sudetes Mts., Eastern Sudetes Mts., Western Beskid Mts., Tatra Mts. (GŁOWACKI et al. 1988, BURAKOWSKI et al. 1992).

\section{Hylastes cunicularius ERICHSON, 1836}

Known from the whole of Poland, except the higher mountains (BURAKOWSKI et al. 1992).

\section{Hylastes linearis ERICHSON, 1836}

Known from: Wielkopolska-Kujawy Lowland, Lower Silesia, Trzebnica Hills, Upper Silesia, Kraków-Wieluń Upland, Małopolska Upland, Western Beskid Mts., Tatra Mts. (BURAKOWSKI et al. 1992). Found in the Lublin Upland (KANIA \& KRÓLIK 2003).

\section{Hylastes opacus ERICHSON, 1836}

Probably inhabits the whole of Poland (BURAKOWSKI et al. 1992).

\section{Hylurgops glabratus (ZETTERSTEDT, 1828)}

Recorded from: Mazovian Lowland, Lower Silesia, Roztocze, Western Sudetes Mts., 
Eastern Sudetes Mts., Western Beskid Mts., Nowy Targ Valley, Eastern Beskid Mts., Bieszczady Mts., Tatra Mts. (BURAKOwSKI et al. 1992).

\section{Hylurgops palliatus (GYLLENHAL, 1813)}

Present in almost the whole of Poland (BURAKOWSKI et al. 1992).

\section{Hylastinus obscurus (MARSHAM, 1802)}

Known from nine regions of Poland (BURAKOWSKI et al. 1992), but all records are over 50 years old.

\section{Hylesinus crenatus (FABRICIUS, 1787)}

Probably present in the whole of Poland (BURAKOWSKI et al. 1992).

\section{Hylesinus wachtli orni FUCHS, 1906 \\ $=$ Lepersinus orni $($ FUCHS1906)}

Recorded from: Pomeranian Lake District, Masurian Lake District, WielkopolskaKujawy Lowland, Białowieża Primeval Forest, Lower Silesia, Trzebnica Hills, Upper Silesia, Western Sudetes Mts., Eastern Sudetes Mts., Western Beskid Mts., Bieszczady Mts. (BURAKOWSKI et al. 1992). According to SCHEDL (1981) the status of this species is unclear.

\section{Hylesinus toranio (DANTHOINE in BERNARD, 1788)}

\section{New records}

Mazovian Lowland: Warsaw (Ursynów) (EC07), 20 IV 1995, 6 exx., leg. T. Mokrzycki (PCTM); Małopolska Upland: Rogów near Koluszki (DC24), 27 VII 1997, 1 ex, leg. J. Borowski (DFPEW); Dąbrówka Nagórna near Radom (EC00), 14 V 2007, 1 ex., leg. M. Miłkowski (PCMM).

Previously recorded from: Pomeranian Lake District, Masurian Lake District, Wielkopolska-Kujawy Lowland, Mazovian Lowland, Białowieża Primeval Forest, Lower Silesia, Kraków-Wieluń Upland, Sandomierz Lowland, Western Beskid Mts., Eastern Beskid Mts., Bieszczady Mts. (BURAKOWSKI et al. 1992).

\section{Hylesinus varius (FABRICIUS, 1775)}

= Lepersinus fraxini (PANZER, 1799)

\section{New record}

Nowy Targ Basin: Zakopane (DV25), 3 V 2001, numerous specimens, leg. T. Mokrzycki (PCTM).

Now recorded from the whole of Poland. 


\section{Kissophagus vicinus (COMOLLI, 1837)}

= Kissophagus hederae (SCHMITT, 1843)

Very rare, known only from the Wielkopolska-Kujawy Lowland, Lower and Upper Silesia (BURAKOWSKI et al. 1992).

\section{Pteleobius kraatzii (EICHHOFF, 1864)}

Collected 50 years ago in Białowieża and in the Warsaw area (BURAKOWSKI et al. 1992).

\section{Pteleobius vittatus (FABRICIUS, 1787)}

New records

Pomeranian Lake District: Bielinek nat. res. (VU46), 24 III 1999, leg. A. Mazur (PCAM); Lower Silesia: Oława (XS64), 05.XI.2000, 5 exx., leg. J. Hilszczański (PCJH); Upper Silesia: Błota (XS74), VIII 2008, numerous specimens, leg. T. Bziuk (PCHS).

Previously recorded from: Wielkopolska-Kujawy Lowland, Mazovian Lowland, Białowieża Primeval Forest, Lower Silesia, Trzebnica Hills, Kraków-Wieluń Upland, Lublin Upland, Eastern Beskid Mts. (BURAKOWSKI et al. 1992).

\section{New record}

\section{Dendroctonus micans (KUGELANN, 1794)}

Małopolska Upland: Zagnańsk (DB74), 4 IV 1994, 1 ex., leg. M. Bidas (PCRC).

Previously recorded from widespread localities in lowland and mountain areas (BURAKOWSKI et al. 1992).

\section{Hylurgus ligniperda (FABRICIUS, 1792)}

Present in the whole of Poland except the Bieszczady Mts., Pieniny Mts. and Tatra Mts. (BURAKOWSKI et al. 1992).

\section{Tomicus minor (HARTIG, 1834)}

Present in the whole of Poland except the Bieszczady Mts., Nowy Targ Valley and Sudetes Mts. (BURAKOWSKI et al. 1992).

\section{Tomicus piniperda (LINNAEUS, 1758)}

Known from the whole of Poland (BURAKOWSKI et al. 1992).

\section{Xylechinus pilosus (RATZEBURG, 1837)}

Recorded from: Baltic Coast, Pomeranian Lake District, Masurian Lake District, 
Wielkopolska-Kujawy Lowland, Trzebnica Hills, Upper Silesia, Roztocze, Western Sudetes Mts., Eastern Sudetes Mts., Western Beskid Mts., Bieszczady Mts., Pieniny Mts., Tatra Mts. (BURAKOWSKI et al. 1992).

\section{Phloeosinus thujae (PERRIS, 1855)}

\section{New records}

Podlasie: Sycze near Siemiatycze (FD31), 19 VI 1995, 2 exx., leg. T. Mokrzycki (PCTM); Białowieża (FD94), 13 VIII 2000, 24 exx. on dying Chamaecyparis lawsoniana, leg. T. Mokrzycki (PCTM); Małopolska Upland: Radom (EB19), 17 VIII 2009, 2 exx., leg. M. Miłkowski (PCMM).

Previously recorded from: Baltic Coast, Pomeranian Lake District, Masurian Lake District, Wielkopolska-Kujawy Lowland, Mazovian Lowland, Podlasie, Lower Silesia, Małopolska Upland, Świętokrzyskie Mts., Lublin Upland, Roztocze, Sandomierz Lowland, Western Beskid Mts., Eastern Beskid Mts., Pieniny Mts. (BURAKOWSKI et al. 1992); has recently become more common as a result of the spread of host plants (Chamaecyparis, Juniperus and Thuja).

\section{Phloeotribus rhododactylus (MARSHAM, 1802)}

\section{New record}

Pomeranian Lake District: Polanów (XV19), V 1997, 3 exx., leg. et cult. J. Borowski (DFPEW).

Previously recorded from: Lower Silesia, Trzebnica Hills, Eastern Sudetes Mts., Western Beskid Mts. (BURAKOWSKI et al. 1992).

\section{Phloeotribus spinulosus (REY 1883)}

Known from both ranges of spruce in Poland (BURAKOWSKI et al. 1992).

\section{New record}

\section{Carphoborus minimus (FABRICIUS, 1798)}

Mazovian Lowland: Sękocin near Warsaw (DC97), XII 2005, 2 exx., leg. et cult. J. Hilszczański (PCJH).

Previously recorded from: Baltic Coast, Wielkopolska-Kujawy Lowland, Mazovian Lowland, Lower Silesia, Trzebnica Hills, Małopolska Upland, Lublin Upland, Roztocze, Western Sudetes Mts., Western Beskid Mts. (BURAKOWSKI et al. 1992). The majority of the records are from the beginning of the twentieth century. 


\section{Polygraphus grandiclava C.G. THOMSON, 1886}

Known from: Kraków-Wieluń Upland, Roztocze, Western Sudetes Mts., Tatra Mts. (BURAKOWSKI et al. 1992).

\section{Polygraphus poligraphus (LINNAEUS, 1758)}

Recorded from whole country except the Sandomierz Lowland and Nowy Targ Valley (BURAKOWSKI et al. 1992).

\section{Polygraphus punctifrons C.G. THOMSON, 1886}

Recorded from: Baltic Coast, Masurian Lake District, Białowieża Primeval Forest, Bieszczady Mts., Pieniny Mts. (BURAKOWSKI et al. 1992), Lower Silesia (MAZUR 1995).

\section{Polygraphus subopacus C.G. THOMSON, 1871}

Known from: Podlasie, Białowieża Primeval Forest, Upper Silesia, Małopolska Upland, Świętokrzyskie Mts., Lublin Upland, Roztocze, Pieniny Mts., Tatra Mts. (BURAKOWSKI et al. 1992).

\section{Pityophthorus carniolicus WICHMANN, 1910}

Recorded for the first time by WANAT \& MOKRZYCKI (2005).

\section{New records}

Mazovian Lowland: Kampinoski Nat. Park (DC59), 3-9 V 2002, 4 exx., leg. R. Ruta (PCRR); Sękocin near Warsaw (DC97), 17 IV 2005, 4 exx., leg. J. Hilszczański (PCJH); Małopolska Upland: Rogów near Koluszki (DC24), 6 V 1994, 12 exx., leg T. Mokrzycki and J. Borowski (PCTM); Pińczów (DA69), 19 V 1993, 6 exx., leg. R. Wolski (PCTM).

\section{Pityophthorus cephalonicae PFEFFER, 1940}

Known only from Greece and Poland (WOOD \& BRIGTH 1992). In Poland recorded in the Pieniny Mts. (NunBERG, 1981). In the Catalogue of Polish Fauna (BURAKOWSKI et al. 1992) Pityophthorus cephalonicae is listed as a synonym of P. pubescens. KNÍŽEK (2011) designated P. cephalonicae as a separate species.

\section{Pityophthorus exsculptus (RATZEBURG, 1837)}

Known from: Baltic Coast, Wielkopolska-Kujawy Lowland, Lower Silesia, Trzebnica Hills, Upper Silesia, Małopolska Upland, Świętokrzyskie Mts., Lublin Upland, Roztocze, Sandomierz Lowland, Western Beskid Mts., Bieszczady Mts. (BURAKOWSKI et al. 1992), but the majority of the data on the species' occurrence are more than 50 years old. 


\section{Pityophthorus glabratus EICHHOFF, 1878}

Recorded from: Wielkopolska-Kujawy Lowland, Białowieża Primeval Forest, Lower Silesia, Świętokrzyskie Mts., Lublin Upland, Sandomierz Lowland, Western Sudetes Mts., Nowy Targ Valley, Pieniny Mts. (BURAKOWSKI et al. 1992); the majority of these records are more than 50 years old.

\section{Pityophthorus knoteki REITTER, 1898}

The exact distribution in Poland is not clear, because of the taxonomical difficulties of separating this species from the closely related Pityophthorus lichtensteinii Ratz. NUNBERG (1981) was unable to identify the species.

\section{Pityophthorus lichtensteinii (RATZEBURG, 1837)}

Known from the whole of Poland except the higher mountains (BURAKOWSKI et al. 1992).

Pityophthorus micrographus micrographus (LINNAEUS, 1758)

Recorded from both ranges of spruce in Poland (BURAKOWSKI et al. 1992).

\section{Pityophthorus morosovi SPESSIVTSEV, 1926}

In Poland known from the southern range of spruce from Niepołomice and the Pieniny Mts. (BURAKOWSKI et al. 1992) and Upper Silesia (GREŃ 2003).

\section{Pityophthorus pityographus pityographus (RATZEBURG, 1837)}

In the whole of Poland except for the Pomeranian Lake District and the higher mountains (BURAKOWSKI et al. 1992).

\section{Pityophthorus pubescens (MARSHAM, 1802)}

Known from: Baltic Coast, Świętokrzyskie Mts., Roztocze, Pieniny Mts. (BURAKOwSKI et al. 1992).

\section{Pityophthorus tragardhi SPESSIVTSEV, 1921}

Recorded from both ranges of spruce in Poland (BURAKOWSKI et al. 1992).

\section{Cryphalus asperatus (GYLLENHAL, 1813)}

= Cryphalus abietis (RATZEBURG, 1837)

Recorded from the whole of Poland except for the Nowy Targ Valley and Trzebnica Hills (BURAKOWSKI et al. 1992). 


\section{Cryphalus intermedius FERRARI, 1867}

New records

Lower Silesia: Henryków for. insp. (XS42), 30 XII 2002, numerous specimens, leg. and cult. A. Mazur (PCAM); Trzebnica Hills: Siemianice near Kępno (YS07), 13 IV 2001, 20 exx., leg. A. Mazur (PCAM); Małopolska Upland: Rogów near Koluszki (DC24), 2 V 1999, 6 exx., leg. T. Mokrzycki (PCTM); Sandomierz Lowland: Tarnobrzeg (EB40), 29 III 2002, 1 ex, leg. R. Cieślak (PCRC).

Previously recorded from: Wielkopolska-Kujawy Lowland, Mazovian Lowland, Upper Silesia, Małopolska Upland, Świętokrzyskie Mts. (BURAKOWSKI et al. 1992).

\section{Cryphalus piceae (RATZEBURG, 1837)}

In Poland recorded throughout the country (BURAKOWSKI et al. 1992).

\section{Cryphalus saltuarius Weise, 1891}

In Poland known from: Białowieża Primeval Forest, Western Sudetes Mts., Eastern Sudetes Mts., Pieniny Mts. (BuraKOWSKI et al. 1992), but all the data on the species' occurrence are more than 50 years old.

\section{New record}

\section{Ernoporicus caucasicus (LINDEMANN, 1876)}

Masurian Lake District: Nowe Kawkowo near Olsztyn (DE46), 9 IX 1995, 8 exx., leg. T. Mokrzycki (PCTM).

Previously recorded from: Pomerania, Wielkopolska-Kujawy Lowland, Silesia (BURAKOWSKI et al. 1992, MOKRZYCKI 1995a)

\section{Ernoporicus fagi (FABRICIUS, 1798)}

Probably inhabits the whole of Poland (BURAKOWSKI et al. 1992).

\section{Ernoporus tiliae (PANZER, 1793)}

Recorded from all parts of Poland except the higher mountains (BURAKOWSKI et al. 1992)

\section{Trypophloeus alni (LINDEMANN, 1875)}

Known from the Bieszczady Mts. and Świętokrzyskie Mts. only (BURAKOWSKI et al. 1992). 
Trypophloeus binodulus (RATZEBURG, 1837)

= Trypophloeus asperatus (GYLLENHAL, 1813)

New record

Lower Silesia: Henryków for. insp. (XS42), 12 IV 1995, 1 ex., leg. and cult. A. Mazur (PCAM).

Previously recorded from: Wielkopolska-Kujawy Lowland, Mazovian Lowland, Białowieża Primeval Forest, Lower Silesia, Kraków-Wieluń Upland, Małopolska Upland, Roztocze, Western Sudetes Mts., Eastern Sudetes Mts., Western Beskid Mts., Eastern Beskid Mts., Bieszczady Mts. (BURAKOWSKI et al. 1992).

\section{Trypophloeus granulatus (RATZEBURG, 1837)}

Recorded from: Masurian Lake District, Wielkopolska-Kujawy Lowland, Mazovian Lowland, Białowieża Primeval Forest, Lower Silesia, Małopolska Upland, Świętokrzyskie Mts. Pieniny Mts. (BURAKOWSKI et al. 1992).

\section{Trypophloeus rybinskii rybinskii REITTER, 1895}

Known from: Wielkopolska-Kujawy Lowland, Mazovian Lowland, Kraków-Wieluń Upland, Sandomierz Lowland, Tatra Mts. (BURAKOWSKI et al. 1992). Collected recently in Góry Pieprzowe nat. res. near Sandomierz (KANIA \& KRÓLIK 2003).

\section{Crypturgus cinereus (HERBST, 1794)}

Polish records from the whole country except the Baltic Coast, Nowy Targ Valley and Tatra Mts. (BURAKOWSKI et al. 1992).

\section{Crypturgus hispidulus C.G. THOMSON, 1870}

Porbably inhabits the whole country with the exception of the higher mountains (BURAKOWSKI et al. 1992).

Crypturgus pusillus (GYLLENHAL, 1813)

Present throughout Poland (BURAKOWSKI et al. 1992).

\section{Crypturgus subcribrosus EGGERS, 1933}

This species was treated as a synonym of Crypturgus cinereus (WOOD \& BRIGTH 1992). According to JORDAL \& KNIŽEK (2007) this is a good species. Because of taxonomical problems the exact distribution is not known. The identification of Crypturgus cinereus in entomological collections needs to be verified. Crypturgus subcribrosus differs from $C$. cinereus by the evidently dull interstriae of the elytra. 
New records

Podlasie: Biebrzański Nat. Park, 23 VII 2004, 3 exx., leg. J. Hilszczański (PCJH); Białowieża Primeval Forest: 26 IX 1954, 12 exx., leg. M. Nunberg (DFPEW); Świętokrzyskie Mts.: Świętokrzyski Nat. Park, 26 IX 1992, (EB03), 4 exx., leg. T. Mokrzycki (PCTM); Western Sudetes Mts.: Sudetes Mountains (Topieliska nat. res.) (XR07), 30 IV 1955, 1 ex., leg. S. Kinelski (DFPEW); Western Beskid Mts.: Kopciowa near Krynica (DV97), 4 VIII 1994, 4 exx., leg. T. Mokrzycki (PCTM).

\section{Dryocoetes alni (GEORG, 1856)}

\section{New records}

Mazovian Lowland: Warsaw (Ursynów) (EC07), 17 IV 1993, 23 exx., leg. T. Mokrzycki (PCTM); Trzebnica Hills: Czeszów (XS59), 15 V 1980, 10 exx., leg. H. Szołtys (PCHS); Małopolska Upland: Rogów near Koluszki (DC24), 22 V 1992, 2 exx, leg. T. Mokrzycki (PCTM).

Previously recorded from: Baltic Coast, Masurian Lake District, Wielkopolska-Kujawy Lowland, Białowieża Primeval Forest, Lower Silesia, Świętokrzyskie Mts., Western Beskid Mts., Eastern Beskid Mts., Tatra Mts. (BURAKOWSKI et al. 1992).

\section{Dryocoetes autographus (RATZEBURG, 1837)}

Recorded throughout Poland (BURAKOWSKI et al. 1992).

\section{Dryocoetes hectographus REITTER, 1913}

More common in the foothills and mountains (BURAKOWSKI et al. 1992).

\section{Dryocoetes villosus villosus (FABRICIUS, 1792)}

\section{New records}

Wielkopolska-Kujawy Lowland: Krajkowo nat. res. (XT38), 6 V 1995, 1 ex., leg. A. Mazur (PCAM); Czeszewski Forest nat. res. (XT67), 27 IV 2007, 1 ex., leg. A. Mazur (PCAM); Upper Silesia: Pokój (XS94), 10 VIII 1998, 1 ex., leg. T. Mokrzycki (PCTM); Sandomierz Lowland: Tarnobrzeg (EB40), 10 XI 2001, 7 exx., leg. R. Cieślak (PCRC).

Previously recorded from: Wielkopolska-Kujawy Lowland, Mazovian Lowland, Podlasie, Białowieża Primeval Forest, Lower Silesia, Trzebnica Hills, Upper Silesia, Kraków-Wieluń Upland, Świętokrzyskie Mts., Roztocze, Sandomierz Lowland, Western Sudetes Mts., Eastern Beskid Mts. (BURAKOWSKI et al. 1992), but the majority of records are from the first half of the twentieth century. 


\section{New record}

\section{Lymantor aceris aceris (LINDEMANN, 1875)}

Małopolska Upland: Rogów near Koluszki (DC24), 17 VIII 2007, 14 exx., leg. J. Borowski (DFPEW).

Previously recorded only from Gdańsk and the Pieniny Mts. (BURAKOWSKI et al. 1992). Recently collected in Upper Silesia (GRZYWOCZ 1996).

\section{New records}

\section{Lymantor coryli (PERRIS, 1855)}

Białowieża Primeval Forest: Białowieża (FD94), 7 VIII 1997, 1 ex., leg. T. Mokrzycki (PCTM); Upper Silesia: Ziemiecice (CA38), 30 IV 1988, 1 ex. and 22 V 1988, 1 ex., leg. J. Hilszczański (PCJH); Małopolska Upland: Gutkowice near Koluszki (DC33), 3 VII 1997, 1 ex., leg. J. Borowski (DFPEW); Rogów near Koluszki (DC24), 2 VII 2006, 1 ex., leg. J. Borowski (DFPEW).

Previously recorded from: Wielkopolska-Kujawy Lowland, Białowieża Primeval Forest, Małopolska Upland, Świętokrzyskie Mts., Roztocze, Sandomierz Lowland, Western Sudetes Mts., Eastern Beskid Mts. Pieniny Mts. (BURAKOWSKI et al. 1992), but the majority of data are very old.

\section{Taphrorychus bicolor (HERBST, 1794)}

Recorded from the whole country except the higher mountains (BURAKOWSKI et al. 1992).

\section{Thamnurgus kaltenbachii (BACH, 1849)}

Known from Gdynia and Puszczykowo near Poznań (BURAKOWSKI et al. 1992). Not recorded for 50 years.

\section{Thamnurgus varipes EICHHOFF, 1878}

Recorded from: Lublin Upland, Roztocze, Western Beskid Mts., Eastern Beskid Mts. (BURAKOWSKI et al. 1992), but the majority of the information comes from the first half of the twentieth century.

\section{New record}

\section{Xylocleptes bispinus (DUFTSCHMID, 1825)}

Mazovian Lowland: Warsaw (Ursynów) (EC07), 3 V 1999, 28 exx., leg. T. Mokrzycki (PCTM). 
Previously recorded from: Baltic Coast, Pomeranian Lake District, WielkopolskaKujawy Lowland, Mazovian Lowland, Lower Silesia, Roztocze, Western Sudetes Mts., Western Beskid Mts., Eastern Beskid Mts. (BURAKOWSKI et al. 1992). In recent years has spread with the host plant Clematis vitalba.

\section{Ips acuminatus (GYLLENHAL, 1827)}

Probably inhabits the whole country (BURAKOWSKI et al. 1992).

Ips amitinus (EICHHOFF, 1872)

Probably occurs throughout the country (BURAKOWSKI et al. 1992).

$$
\text { Ips cembrae (HErr, 1836) }
$$

Recorded from: Pomeranian Lake District, Lower Silesia, Upper Silesia, KrakówWieluń Upland, Małopolska Upland, Świętokrzyskie Mts., Sandomierz Lowland, Western Sudetes Mts., Eastern Sudetes Mts., Western Beskid Mts., Tatra Mts. (BuRAKOWSKI et al. 1992).

Ips duplicatus (C.R. SAHLBERG, 1836)

Recorded in both ranges of spruce in Poland (BURAKOWSKI et al. 1992).

$$
\text { Ips sexdentatus (BOERNER, 1776) }
$$

Recorded from the whole country (BURAKOWSKI et al. 1992).

$$
\text { Ips typographus (LINNAEUS, 1758) }
$$

Reported from the whole country (BURAKOWSKI et al. 1992).

\section{Orthotomicus laricis (FABRICIUS, 1792)}

Reported from the whole of Poland except the higher mountains (BURAKOWSKI et al. 1992).

\section{Orthotomicus longicollis (GYLLENHAL, 1827)}

In Poland recorded from: Masurian Lake District, Wielkopolska-Kujawy Lowland, Mazovian Lowland, Podlasie, Białowieża Primeval Forest, Lower Silesia, Świętokrzyskie Mts., Lublin Upland, Sandomierz Lowland (BURAKOWSKI ET AL. 1992). The majority of data on the distribution of this species are from before 1960. There are recent records from the Białowieża Primeval Forest (MOKRZYCKI 1995b, 2004).

\section{Orthotomicus proximus (EICHHOFF, 1868)}

Known from almost the whole of the country (BURAKOWSKI et al. 1992). 


\section{Orthotomicus starki SPESSIVTSEV, 1926}

Found only in the Białowieża Primeval Forest (BURAKOWSKI et al. 1992, MOKRZYCKI 1995b).

Orthotomicus suturalis (GYLLENHAL, 1827)

Recorded in the whole of the country except the higher mountains (BURAKOWSKI et al. 1992).

\section{Pityogenes bidentatus (HERBST, 1784)}

Recorded from the whole of Poland (BURAKOWSKI et al. 1992).

\section{New record}

\section{Pityogenes bistridentatus (EICHHOFF, 1878)}

Western Sudetes Mts.: Karkonoski National Park (near Karpacz), 19 V-1 VI 2000, 22 exx. in pheromone traps, leg. W. Janiszewski and A. Kolk (PCTM).

Previously recorded from: Wielkopolska-Kujawy Lowland, Lower Silesia, Upper Silesia, Świętokrzyskie Mts., Lublin Upland, Roztocze, Sandomierz Lowland, Western Sudetes Mts., Western Beskid Mts., Nowy Targ Valley, Tatra Mts. (BURAKOwsKI et al. 1992).

\section{Pityogenes chalcographus (LINNAEUS, 1760)}

Reported from the whole of Poland (BURAKOWSKI et al. 1992).

\section{Pityogenes irkutensis monacensis FUCHS, 1911}

Known from: Mazovian Lowland, Podlasie, Białowieża Primeval Forest, Świętokrzyskie Mts., Sandomierz Lowland (BURAKOWSKI et al. 1992). The majority of data on the species' distribution are more than 50 years old.

\section{Pityogenes quadridenes (HARTIG, 1834)}

Recorded in almost the whole of Poland (BURAKOWSKI et al. 1992).

\section{Pityogenes saalasi EgGERS, 1914}

Known only from the Białowieża Primeval Forest (BURAKOwSKI et al. 1992); not recorded for over fifty years.

\section{Pityogenes trepanatus (NöRDLINGER, 1848)}

In Poland recorded from: Pomeranian Lake District, Wielkopolska-Kujawy Lowland, 
Mazovian Lowland, Podlasie, Białowieża Primeval Forest, Lower Silesia, Małopolska Upland, Świętokrzyskie Mts., Lublin Upland, Sandomierz Lowland, Western Sudetes Mts., Eastern Sudetes Mts. (BURAKOWSKI et al. 1992) and Baltic Coast (MOKRZYCKI 1995a).

\section{Pityokteines curvidens (GERMAR, 1824)}

Throughout the range of fir in Poland (BURAKOWSKI et al. 1992).

\section{Pityokteines spinidens (REITTER, 1895)}

Throughout the range of fir in Poland (BURAKOWSKI et al. 1992).

Pityokteines vorontzowi (JACOBSON, 1896)

Throughout the range of fir in Poland (BURAKOWSKI et al. 1992).

\section{New record}

\section{Scolytus carpini (RATZEBURG, 1837)}

Wielkopolska-Kujawy Lowland: Czeszewski Forest nat. res. (XT67), 27 IV 2007, 13 exx., leg. A. Mazur (PCAM).

Previously recorded from: Pomeranian Lake District, Wielkopolska-Kujawy Lowland, Mazovian Lowland, Białowieża Primeval Forest, Lower Silesia, Trzebnica Hills, Upper Silesia, Kraków-Wieluń Upland, Lublin Upland, Eastern Sudetes Mts., Eastern Beskid Mts., Pieniny Mts. (BURAKOWSKI et al. 1992).

\section{Scolytus ensifer EICHНOFF, 1881}

New records

Pomeranian Lake District: Bielinek nat. res. (VU46), 24 III 1999, 1 ex., leg. A. Mazur (PCAM); Mazovian Lowland: Kozienicka Forest (EC21), 17-21 III 2004, 3 exx., leg. et cult. M. Miłkowski (PCMM); Krakowiany (DC86), 15 I 2005, cult. em. II-III. 2005, 5 exx., leg. J. Hilszczański (PCJH); Upper Silesia: Segiet nat. res. (CA48), 2 VII 1997, 4 exx., leg. H. Szołtys (PCHS).

Previously recorded from: Pomeranian Lake District, Wielkopolska-Kujawy Lowland, Mazovian Lowland, Lower Silesia, Trzebnica Hills, Kraków-Wieluń Upland, Małopolska Upland, Lublin Upland, Sandomierz Lowland (BURAKOWSKI et al. 1992).

\section{Scolytus intricatus (RATZEBURG, 1837)}

Reported from the whole country except the higher mountains (BURAKOWSKI et al. 1992). 


\section{Scolytus kirschii kirschii SKALITZKY, 1876}

Known from: Wielkopolska-Kujawy Lowland, Podlasie, Białowieża Primeval Forest, Lower Silesia, Upper Silesia, Kraków-Wieluń Upland, Lublin Upland (BURAKOWSKI et al. 1992). The majority of data on the distribution of this species are more than 30 years old.

\section{Scolytus laevis CHAPUIS, 1869}

New records

Mazovian Lowland: Krakowiany (DC86), VI-IX. 2005, window trunk trap, 2 exx., leg. et cult. J. Hilszczański (PCJH); Eastern Beskid Mts.: Rymanów for. insp. (EV 68), 17 VI 2002, 11 exx, leg. J. Borowski (PCTM).

Previously recorded from the Wielkopolsko-Kujawska Lowland, Podlasie, Lublin Upland and Pieniny Mts. (BURAKOWSKI et al. 1992). In recent years the species has extended its range of occurrence. Recently recorded in the Białowieża Primeval Forest (MOKRZYCKI 2004).

\section{Scolytus mali (BECHSTEIN, 1805)}

Recorded in almost the whole of Poland (BURAKOWSKI et al. 1992).

Scolytus multistriatus (MARSHAM, 1802)

Recorded in the whole of Poland except the higher mountains (BURAKOWSKI et al. 1992).

\section{New records}

\section{Scolytus pygmaeus (FABRICIUS, 1787)}

Mazovian Lowland: Krakowiany (DC86), 15 I 2005, cult. em. II-III.2005, 2 exx., leg. J. Hilszczański (PCJH); Lower Silesia: Błota (XS74), 12 exx., cult. VIII 2008, leg. T. Bziuk (PCHS); Małopolska Upland: Pińczów (DA69), 2 VII 1995, 2 exx., leg. T. Mokrzycki (PCTM); Skorocice nat. res. (DA78), 14 VII 1996, 2 exx., leg. H. Szołtys (PCHS).

Previously recorded from: Baltic Coast, Pomeranian Lake District, Masurian Lake District, Wielkopolska-Kujawy Lowland, Mazovian Lowland, Podlasie, Białowieża Primeval Forest, Lower Silesia, Trzebnica Hills, Upper Silesia, Kraków-WieluńUpland, Małopolska Upland, Lublin Upland, Sandomierz Lowland, Eastern Sudetes Mts., Eastern Beskid Mts., Pieniny Mts. (BURAKOWSKI et al. 1992).

\section{Scolytus ratzeburgii E.W. JANSON, 1856}

Reported from almost the whole country (BURAKOWSKI et al. 1992). 


\section{Scolytus rugulosus (P.W.J. MÜLLER, 1818)}

Inhabits almost the whole country (BURAKOWSKI et al. 1992).

Scolytus scolytus (FABRICIUS, 1775)

Recorded from almost the whole country (BURAKOWSKI et al. 1992).

Anisandrus dispar (FABRICIUS, 1792)

= Xyleborus dispar (FABRICIUS, 1792)

Recorded from the whole country with the exception of Tte Nowy Targ Valley, Pieniny Mts. and Tatra Mts. (BURAKOWSKI et al. 1992).

\section{Xyleborinus attenuatus (BLANDFORD, 1894) \\ = Xyleborinus alni (NIISIMA, 1909)}

Distributed in Japan and on Siberia (WOOD \& BRIGTH 1992). The distribution in Europe is not clear, because of confusion with Xyleborinus saxesenii. Evident localities are situated in Austria, the Czech Republic, Germany, Slovakia and Switzerland (KNÍŽEK, 1988, 2011).

In Poland recorded from Upper Silesia (BURAKOWSKI et al. 2000) but is probably much more widespread.

\section{New records}

Mazovian Lowland: Zalesie Górne near Warsaw (EC06), 3 III 1968, 2 exx., leg. S. Mazur (DFPEW); Warsaw (Ursynów) (EC07), 23 IV 1994, 4 exx., leg. T. Mokrzycki (PCTM); Podlasie: Knyszyńka Forest, 16 VI 2003, 1 ex., leg. J. Hilszczański (PCJH); Białowieska Primeval Forest: (FD94), 26 IV 1994, 5 exx., leg. T. Mokrzycki (PCTM); Upper Silesia: Brynek (CA39), 14 II 1999, 2 exx., leg. H. Szołtys (PCTM); Ruda Śląska (CA46), IV 2007, leg T. Bziuk (PCHS) and Stare Tarnowice (CA48), 5 V 2007, leg. H. Szołtys (PCHS); Świętokrzyskie Mts.: Świętokrzyski Nat. Park, 11 V 1994, (EB03), 5 exx., leg. T. Mokrzycki (PCTM); Eastern Beskid Mts.: Jawornik near Dębica (EA14), 23 IV 1993, 1 ex., leg. J. Hilszczański (PCJH).

\section{Xyleborinus saxesenii (RATZEBURG, 1837)}

Probably occurs throughout the country.

\section{Xyleborus cryptographus (RATZEBURG, 1837)}

\section{New records}

Mazovian Lowland: Warsaw (Ursynów) (EC07), 26 IV 2011, numerous specimens, leg. T. MOKRZYCKI (PCTM); Małopolska Upland: Radom (Kapturski Forest) (EB 09), 23 III 1994, 5 exx., leg. M. Miłkowski (PCMM). 
Previously recorded from: Pomeranian Lake District, Masurian Lake District, Wielkopolska-Kujawy Lowland, Mazovian Lowland, Białowieża Primeval Forest, Lower Silesia, Upper Silesia, Kraków-Wieluń Upland, Małopolska Upland, Świętokrzyskie Mts., Lublin Upland, Roztocze, Eastern Beskid Mts. (BURAKOWSKI et al. 1992).

\section{New record}

\section{Xyleborus dryographus (RATZEBURG, 1837)}

Lower Silesia: Błota near Brzeg (XS74), 7 VI 2008, numerous specimens, leg T. Bziuk (PCHS).

Previously recorded from: Masurian Lake District, Wielkopolska-Kujawy Lowland, Mazovian Lowland, Lower Silesia, Trzebnica Hills, Upper Silesia, Kraków-Wieluń Upland, Świętokrzyskie Mts., Lublin Upland, Roztocze, Eastern Beskid Mts. (BURAKOWSKI et al. 1992, MOKRZYCKI et al. 2008).

\section{Xyleborus eurygraphus (RATZEBURG, 1837)}

Known from: Lower Silesia, Trzebnica Hills, Lublin Upland, Roztocze (BURAKOWSKI et al. 1992), but all the data regarding its occurrence are more than 50 years old and the presence of the species in the country should be supported by new observations.

\section{Xyleborus monographus (FABRICIUS, 1792)}

Recorded from the lowlands, less often from the on mountain foothills; not reported from the Carpathian and Sudetes Mts. (BURAKOwSKI et al. 1992).

\section{Xyleborus pfeilii (RATZEBURG, 1837)}

Known from: Wielkopolska-Kujawy Lowland, Mazovian Lowland, Upper Silesia, Roztocze, Pieniny Mts. (BURAKOWSKI et al. 1992); All data on the species' occurrence come from before 1960, so the presence of the species in the country should be supported by new observations.

\section{Xylosandrus germanus (BLANDFORD, 1894)}

Introduced from eastern Asia to Europe and North America. Known from Austria, Belgium, France, Germany, Switzerland, Italy, Canada and USA (WOOD \& BRIGHT 1992, KNÍŽEK 2011). First recorded in Poland by WANAT \& MOKRZYCKI (2005). Similar to Xyleborus dispar, from which it differs by the smaller body size $(2.0-2.3 \mathrm{~mm})$ and the wellseparated procoxae.

New records

Baltic Coast: Międzyzdroje (VV67), 3 VI 1998, 7 exx., leg. H. Szołtys (PCHS, PCTM); 
Mazovian Lowland: Kozienicka Forest (Krępiec nat. res.) (EC40), 18 VI 2006, 3 exx., on Viscum album austriacum, leg. M. Miłkowski (PCMM, PCTM); Kampinoski National Park (DC89), 10 V 2010, 2 exx., leg. D. Marczak (PCDM); Upper Silesia: Murcki (CA66), 17 IV 2011, 60 exx., leg. T. Bziuk (PCHS). A few records from the Baltic Coast, Mazovian Lowland and Upper Silesia suggest a broader distribution.

\section{Trypodendron domesticum (LINNAEUS, 1758)}

Known from the whole country with the exception of the higher mountains (BURAKOWSKI et al. 1992).

\section{Trypodendron lineatum (A.G. OLIVIER, 1795)}

Known from the whole country with the exception of the Trzebnica Hills (BURAKOWSKI et al. 1992).

\section{Trypodendron signatum (FABRICIUS, 1792)}

Recorded from almost the whole country; rarely in the mountains (BURAKOWSKI et al. 1992).

\section{SPECIES EXCLUDED FROM THE CHECKLIST}

\section{Hylastes plumbeus BLANDFORD, 1894}

Reported from Poland by KNÍŽEK (2011). The only mention of its occurrence in Białowieża Forest from 50 years ago was not subsequently confirmed (BURAKOWSKI et al. 1992).

\section{Phloeosinus aubei (PERRIS, 1855)}

Reported from Poland by KNÍŽEK (2011), but not included in the Catalogue of Polish Fauna (BURAKOWSKI et al. 1992, 2000).

\section{Carphoborus cholodkovskyi SPESSIVTSEV, 1916}

Recorded only once from the Białowieża Forest by KARPIŃSKI (1933). Later not found. This locality now lies in the Belorussian part of the Białowieża Forest, so the occurrence of the species in Poland is not confirmed.

\section{Gnathotrichus materiarius (FITCH, 1855)}

Reported from Poland by KNížEK (2011), but not included in the Catalogue of Polish Fauna (BURAKOWSKI et al. 1992, 2000). 


\section{Orthotomicus erosus (WoLLASTON, 1857)}

Existing information about this species' occurrence in Poland apply to the related Orthotomicus proximus.

\section{Orthotomicus mannsfeldi (WACHTL, 1880)}

Reported from Poland by KNížEK (2011), but not included in the Catalogue of Polish Fauna (BURAKOWSKI et al. 1992, 2000).

\section{Scolytus sulcifrons REY, 1883}

Reported from Poland by KNÍŽEK (2011), but not included in the Catalogue of Polish Fauna (BURAKOWSKI et al. 1992, 2000).

\section{Trypodendron laeve EGGERS 1939}

Reported from Poland by KNÍŽEK (2011), but BURAKOWSKI et al. (2000) do not include this species among the Polish fauna.

\section{SPECIES REGULARLY INTRODUCED TO POLAND}

\section{Hypothenemus hampei (FERRARI, 1867)}

Widespread in the tropical zone, on coffee plantations. Introduced to Poland together with seeds of Coffea arabica and C. robusta (BURAKOWSKI et al. 1992).

\section{Coccotrypes carpophagus (HORNUNG, 1842)}

$=$ C. dactyliperda (FABRICIUS, 1801)

Known from the whole of the tropical and equatorial regions (WOOD \& BRIGHT 1992). Introduced to Poland with the seeds of various species from the family Arecaceae (BURAKOWSKI et al. 1992).

\section{Xyleborus perforans (WOLLASTON, 1857)}

Known from the tropical and equatorial zones; the most northerly range limit known is Japan (WOOD \& BRIGHT 1992). Introduced to Poland with tropical timber (BURAKOWSKI et al. 1992).

\section{DISCUSSION}

Excluding Carphoborus cholodkovskyi, the updated Polish checklist of the Scolytinae comprises 110 species and the Platypodinae - one species.

The biology and occurrence of the tree-damaging species are well-known, but this is not the case with the remaining species. Most recent Polish records of Hylastinus obscurus, 
Pteleobius kraatzii, Pityophthorus exsculptus, Cryphalus saltuarius, Thamnurgus kaltenbachii, T. varipes, Pityogenes irkutensis monacensis, P. saalasi, Xyleborus eurygraphus and $X$. pfeilii are 50 and more years old. The occurrence of these ten rare species in Poland must be confirmed by new findings.

On the list of endangered and threatened animals in Poland, the status of Kissophagus vicinus, Lymantor aceris aceris, Thamnurgus varipes and Orthotomicus starki is "Least Concern"; that of Dendroctonus micans, Polygraphus grandiclava, Trypophloeus rybinskii rybinskii and Xyleborus pfeilii is "Vulnerable"; and that of Phloeotribus rhododactylus, Cryphalus saltuarius, Ernoporicus caucasicus, Trypophloeus alni, Thamnurgus kaltenbachii and Xyleborus eurygraphus is "Data Deficient" (GŁOWACIŃSKI 2002).

\section{REFERENCES}

Burakowski B., Mroczkowski M., Stefańska J. 1992. Ryjkowcowate prócz ryjkowców Curculionoidea prócz Curculionidae. Chrząszcze - Coleoptera. Katalog Fauny Polski. Part 23, vol. 18, Warszawa, $324 \mathrm{pp}$.

Burakowski B., Mroczkowski M., Stefańska J. 2000. Uzupełnienia tomów 2-21. Chrząszcze Coleoptera. Katalog Fauny Polski. Part 23, vol. 22, Warszawa, 252 pp.

GŁOWACIŃSKi Z. (ed.) 2002. Polska lista zwierząt ginących i zagrożonych w Polsce. Zakład Ochrony Przyrody i Zasobów Naturalnych PAN, Kraków, 155 pp.

GŁOWACKi A., Michalski J., KonCA B. 1988. Nowe stanowiska Hylastes brunneus ER. (Coleoptera, Scolitydae) w Polsce. Parki Narodowe i Rezerwaty Przyrody 2: 57-59.

Greń C. 2003. Pierwsze stanowisko Pityophthorus morosovi SPESSIVtSefF, 1926 (Coleoptera: Scolytidae) i Ernobius longicornis (STURM, 1837) na Górnym Śląsku (Coleoptera: Anobidae). Acta Entomologica Silesiana 9-10: 8.

GRZYwOCZ J. 1996. Lymantor aceris (LinDEMANN, 1875) nowy dla fauny Śląska gatunek kornika (Coleoptera: Scolytidae). Acta Entomologica Silesiana 4: 24.

JoRDAL B.H., KNIŽEK M. 2007. Resurrection of Crypturgus subcribrosus EGGERS 1933 stat. n., and its close phylogenetic relationship to Nearctic Crypturgus (Coleoptera, Scolytinae). Zootaxa 1606: 41-50.

KANIA J., KRÓLIK R. 2003. Nowe stanowiska Hylastes linearis ERICHSON, 1836 i Trypophloeus rybinskii (ReITter, 1895) w Polsce (Coleoptera: Scolytidae). Acta Entomologica Silesiana 9-10: 86.

KARPIŃSKI J.J. 1933. Fauna korników Puszczy Białowieskiej na tle występujących w Puszczy drzewostanów. Rozprawy i sprawozdania. Zakład Doświadczalny Lasów Państwowych, 68 pp.

KNížEK M. 1988. Faunistic record from Czechoslovakia: Coleoptera, Scolytidae, Xyleborinus alni NIIJIMA. Acta Entomologica Bohemoslovaca 85: 396.

KNížEK, M. 2011. Scolytinae. [In:] LÖBl I., SMetanA A. (ed.). Catalogue of Palaearctic Coleoptera. Vol. 7, Curculionoidea I, 373 pp.

Mazur A. 1995. O występowaniu Polygraphus punctifrons ThOMs (Col., Scolytidae) na Przedgórzu Sudeckim. Wiadomości Entomologiczne 14: 149-152.

MoKRZYCKI T. 1995a. Nowe stanowiska chrząszczy z rodziny Scolytidae (Coleoptera) w Polsce. Wiadomości Entomologiczne 14: 126.

MOKRZYCKI T. 1995b. Zmiany fauny korników (Coleoptera: Scolytidae) jako element monitoringu ekologicznego na terenie północno-wschodniej Polski. Prace Instytutu Badawczego Leśnictwa 
A(796), 149-160.

MOKRZYCKI T. 2004. Kornikowate (Coleoptera, Scolytidae) jako element monitoringu ekologicznego w Puszczy Białowieskiej. Leśne Prace Badawcze 4: 51-66.

MoKRZYCKI T., BYK A., BOROWSKI J. 2008. Rzadkie i reliktowe saproksyliczne chrząszcze (Coleoptera) starych dębów Rogalińskiego Parku Krajobrazowego. Parki Narodowe i Rezerwaty Przyrody 27: 43-56.

NunBerg M. 1960. Wiadomości o występowaniu niektórych korników (Col. Scolytidae) na ziemiach Polski. Polskie Pismo Entomologiczne 30: 153-162.

NunBeRG M. 1981. Korniki - Scolytidae, wyrynniki - Platypodidae. Klucze do oznaczania owadów Polski. Chrząszcze - Coleoptera. XIX (99-100), 115 pp.

SchedL K.E. 1972. Monographie der Familie Platypodidae. Dr. W. JunK N.V. - Den Haag, 322 pp.

SCHEDL K.E. 1981. Scolytidae (Borken- und Ambrosiakäfer). [In:] Freude, H., HARDE, K. W., LOHSE, G.A. (eds). Die Käfer Mitteleuropas 10, 99 pp.

Wanat M., MokrzyCKi T. 2005. A new checklist of the weevils of Poland. Genus 16: 69-117.

WooD S.L. 1986. A Reclassification of the Genera of Scolytidae (Coleoptera). Great Basin Naturalist Memoirs 10, $126 \mathrm{pp}$.

Wood S.L., Bright D.E. 1992. A Catalog of Scolytidae and Platypodidae (Coleoptera), Part 2: Taxonomic Index. Great Basin Naturalist Memoirs 13, 1553 pp.

Received: April 28, 2011

Accepted: May 31, 2011 